\title{
d-Primitive Words and Contextual Grammars
}

\author{
Tetsuo MORIYA ${ }^{\dagger a)}$ and Itaru KATAOKA ${ }^{\dagger}$, Members
}

SUMMARY In this paper we study the ploblem whether the language $D(1)$ of all d-primitive words can be generated by a contextual grammar. It is proved that $D(1)$ can be generated neither by an external contextual grammar nor by an internal contextual grammar, and that it can be generated by a total contextual grammar with choice.

key words: formal language, primitive word, non-overlapping word, $d$ primitive word, context-free, Marcus contextual grammar and language

\section{Introduction}

Primitivity of words is a very important concept, and has played an important role in the theory of codes and relative aspects of combinatorics of words. It is well-known conjecture of Domosi et.al that the language $\mathrm{Q}$ of all primitive words is not context-free [2]. This conjecture has not yet been proved so far.(See [3] and [6]) However, it has been proved that $\mathrm{Q}$ can be generated by some types of contextual grammars [4].

On the other hand, the language $\mathrm{D}(1)$ of all d-primitive words, a proper subset of $\mathrm{Q}$, is important as well as $\mathrm{Q}$, and a lot of studies have been done on $\mathrm{D}(1)$ (See, for example, [1], [5], [7], [11]). The problem whether or not $\mathrm{D}(1)$ is contextfree was raised in [8], and this is also still unsolved. It is a natural and interesting question whether or not $\mathrm{D}(1)$ can be generated by a contextual grammar.

In this paper, we consider the very theme. It is proved that $\mathrm{D}(1)$ can be generated neither by an external contextual grammar nor by an internal contextual grammar, and that it can be generated by a total contextual grammar with choice.

\section{Preliminaries}

Let $\Sigma$ be an alphabet consisting of at least two letters. $\Sigma^{*}$ denotes the free moniod generated by $\Sigma$, that is, the set of all finite words over $\Sigma$, including the empty word $\epsilon$, and $\Sigma^{+}=$ $\Sigma^{*}-\{\epsilon\}$. For $w$ in $\Sigma^{*},|w|$ denotes the length of $w$. A language over $\Sigma$ is a set $L \subseteq \Sigma^{*}$. If $u=v x w$ for some $v, w \in \Sigma^{*}, x$ is called an infix or a factor of $u$.

A nonempty word $u$ is called a primitive word if $u=f^{n}$, for some $f \in \Sigma^{+}$, implies that $n=1$ and $u=f^{n}$. Let $Q$ be the set of all primitive words over $\Sigma$. A nonempty word $u$ is a non-overlapping word if $u=v x=y v$ for some $x, y \in \Sigma^{+}$

\footnotetext{
Manuscript received May 17, 2012.

${ }^{\dagger}$ The authors are with School of Science and Engineering, Kokushikan University, Tokyo, 154-8515 Japan.

a)E-mail: moriya@kokushikan.ac.jp

DOI: 10.1587/transinf.E95.D.2710
}

always implies that $v=\epsilon$. Let $D(1)$ be the set of all nonoverlapping words over $\Sigma$. A word in $D(1)$ is also called a $d$-primitive word (See [1] and [10]).

$A$ (Marcus) contextual grammar with choice is a structure $G=(\Sigma, A, C, \psi)$ where $\Sigma$ is an alphabet, $A$ is a finite subset of $\Sigma^{*}$, i.e. the set of axioms, $C$ is a finite subset of $\Sigma^{*} \times \Sigma^{*}$, i.e.the set of contexts, and $\psi: \Sigma^{*} \rightarrow 2^{C}$ is the choice function. If $\psi(x)=C$ hold for every $x \in \Sigma^{*}$, then we say that $G$ is a (Marcus) contextual grammar without choice and then we omit $\psi$ sometimes. We define two relations on $\Sigma^{*}$ : for any $x \in \Sigma^{*}$, we write $x \Rightarrow_{e x} y$ if and only if $y=u x v$ for a context $(u, v)$ in $\psi(x), x \Rightarrow_{\text {in }} y$ if and only if $x=x_{1} x_{2} x_{3}, y=x_{1} u x_{2} v x_{3}$ for some $(u, v) \in \psi\left(x_{2}\right)$. By $x \Rightarrow_{e x}^{*}$ $y$ and $x \Rightarrow{ }_{i n}^{*} y$, we denote the reflexive and transitive closure of these relations and $L_{\alpha}(G)=\left\{x \in \Sigma^{*} \mid w \Rightarrow_{\alpha}^{*} x, w \in A\right\}$ for $\alpha \in\{e x, i n\} . \mathrm{By} \Rightarrow_{e x}^{+}$and $\Rightarrow_{i n}^{+}$, we denote the transitive closure of these relations. Then $L_{e x}(G)$ is the (Marcus) external contextual language (with or without choice) generated by $G$, and similarly, $L_{i n}(G)$ is the (Marcus) internal contextual language (with or without choice) generated by $G$.

A total contextual grammar with choice is a structure $G=(\Sigma, A, C, \Psi)$ where $\Sigma$ is an alphabet, $A$ is a finite language over $\Sigma, C$ is a finite subset of $\Sigma^{*} \times \Sigma^{*}$ and $\Psi: \Sigma^{*} \times \Sigma^{*} \times \Sigma^{*} \rightarrow 2^{C}$. The generated language is $L(G)=\left\{x \in \Sigma^{*} \mid w \Rightarrow^{*} x, w \in A\right\}$ where $\Rightarrow^{*}$ is the reflexive and transitive closure of the relation on $\Sigma^{*}$ defined by $x \Rightarrow y$ if and only if $x=x_{1} x_{2} x_{3}, y=x_{1} u x_{2} v x_{3}, x_{1}, x_{2}, x_{3} \in \Sigma^{*}$ for a context $(u, v) \in \Psi\left(x_{1}, x_{2}, x_{3}\right)$.

We denote by ECC(resp.EC) the family of external contextual languages with choice (without choice), by $I C C$ (resp.IC) the family of internal contextual languages with choice (without choice), and by $T C$ the family of total contextual languages with choice.

Proposition 1 ([9]): (1)EC $\subset E C C$.

(2) $I C \subset I C C$.

(3) $E C C \subset T C$, and $I C C \subset T C$.::

\section{3. d-Primitive Words and Contextual Grammars}

First we prove that $D(1)$ is not an internal contextual language.

Fact 2: Let $n \geq 0$.

(1) $1 \leq l, m \leq k, a^{m} b^{k}\left(a^{k} b^{k}\right)^{n} a^{k} b^{l}$ is not in $D(1)$.

(2) For $1 \leq k<m, a^{m} b^{k}\left(a^{k} b^{k}\right)^{n} a^{k} b^{k}$ is in $D(1)$. 
Proposition 3: The language $\mathrm{D}(1)$ of all d-primitive words is not an internal contextual language with choice.

\section{Proof.}

Suppose that there exists a $G=(\Sigma, A, C, \psi)$ with $D(1)=$ $L_{\text {in }}(G)$. Then there exist $u, v, w \in \Sigma^{+}$such that $u v \in \Sigma^{+}$and $(u, v) \in \psi(w)$. Let $|u w v|=k$. Suppose that the last letter of $u w v$ be $b$, and $b \neq a \in \Sigma$. By the assumption, it is obvious that $x=a^{2 k} b w a^{2 k} b u v w$ is in $D(1)$.

We have that for $x=a^{2 k} b w a^{2 k} b u v w$,

$$
x \Rightarrow_{\text {in }} a^{2 k} \text { buwva } a^{2 k} \text { buwv }=y \text {. }
$$

However, this contradicts the assumption $D(1)=$ $L_{\text {in }}(G)$ since $y$ is not in $D(1)$. Thus the results holds. ::

Corollary 4: The language $\mathrm{D}(1)$ of all d-primitive words is not an internal contextual language without choice.

Next we prove that $D(1)$ is not an external contextual language.

Proposition 5: The language $\mathrm{D}(1)$ of all d-primitive words is not an external contextual language with choice.

Proof. Suppose that there exists a $G=(\Sigma, A, C, \psi)$ with $D(1)=L_{e x}(G)$.

Let $k=\max \left\{|u|+|v|+|\alpha| \mid(u, v) \in \psi(w), w \in \Sigma^{*}, \alpha \in A\right\}$ +1 . Consider the word $x=a^{k+1} b^{k} a^{k} b^{k}$. By Fact 1 (2), $x$ is in $D(1)$. There exist $\alpha \in A, z_{1}, \ldots, z_{i} \in D(1)$ such that $\alpha \Rightarrow_{e x} z_{1} \Rightarrow_{e x} \ldots \Rightarrow_{e x} z_{i} \Rightarrow x$.

Note that $z_{1}, \ldots, z_{i}$ are factors of $x$, and that the length increasable by one step of the derivation is less than $k$.

By the assumption of $k$, we have that $x=y_{1} z_{1} y_{2}$ for some $y_{1}, y_{2} \in \Sigma^{+}$.

(Case 1) $x=a^{t} z_{1} b^{s} a^{k} b^{k}$ for some $s, t \geq 1$, that is, $z_{1}=a^{m} b^{l}$, where $t+m=l+s=k$.

There exist integers $l_{1}, l_{2}, m_{1}, m_{2}, m_{3}$ such that $a^{m} b^{l} \Rightarrow_{e x}^{*} a^{m_{1}} b^{l_{1}} \Rightarrow_{e x} a^{m_{2}} b^{k} a^{m_{3}} \Rightarrow_{e x}^{*} x$

with $1 \leq m \leq m_{1} \leq m_{2}<k$, and $1 \leq l \leq l_{1}<k, 1 \leq m_{3}<k$. This is a contradiction since $a^{m_{2}} b^{k} a^{m_{3}} \notin D(1)$.

(Case 2) $x=a^{k} b^{s} z_{1} a^{t} b^{k}$ for some $s, t \geq 1$, that is, $z_{1}=b^{l} a^{m}$, where $s+l=m+t=k$.

There exist integers $l_{1}, l_{2}, m_{1}, m_{2}$ such that $b^{l} a^{m} \Rightarrow_{e x}^{*} b^{l_{1}} a^{m_{1}} \Rightarrow_{e x} a^{m_{2}} b^{k} a^{k} b^{l_{2}} \Rightarrow_{e x}^{*} x$

with $1 \leq m \leq m_{1}<k, 1 \leq l \leq l_{1}<k$, and $1 \leq l_{2}, m_{2}<k$. This is a contradiction since $a^{m_{2}} b^{k} a^{k} b^{l_{2}} \notin D(1)$ by Fact $1(1)$. (Case 3) $x=a^{k} b^{k} a^{s} z_{1} b^{t}$ for some $s, t \geq 1$, that is,

$z_{1}=a^{m} b^{l}$, where $s+m=l+t=k$.

There exist integers $l_{1}, l_{2}, l_{3}, m_{1}$ such that $a^{m} b^{l} \Rightarrow_{e x}^{*} a^{m_{1}} b^{l_{1}} \Rightarrow_{e x} b^{l_{2}} a^{k} b^{l_{3}} \Rightarrow_{e x}^{*} x$ with $1 \leq l \leq l_{1} \leq l_{3}<k, 1 \leq m \leq m_{1}<k$, and $1 \leq l_{2}<k$.

This is a contradiction since $b^{l_{2}} a^{k} b^{l_{3}} \notin D(1)$.:

Corollary 6: The language $\mathrm{D}(1)$ of all d-primitive words is not an external contextual language without choice.

$\because$

Last we prove that $D(1)$ can be generated by a total contextual grammar with choice.

Lemma 7: Let $|x|=|y|$ for $x, y \in \Sigma^{+}$. If $x y \notin D(1)$, then $x a y \notin D(1)$ and $x a b y \notin D(1)$ where $a, b \in \Sigma$. ::

Theorem 8: The language $D(1)$ is a total contextual language with choice.

Proof. Define $G=(\Sigma, A, C, \Psi)$ as follows. Let $A=\Sigma, C$ $=\left\{(u, v)\left|u v \in \Sigma^{+},\right| u v \mid \leq 2\right\}$ and moreover $\Psi\left(x_{1}, x_{2}, x_{3}\right)=$ $\left\{(u, v) \in C \mid x_{1} u x_{2} v x_{3} \in D(1)\right\}$.

By the definition of $G$, it is obvious that $L(G) \subseteq D(1)$. Next we prove that $D(1) \subseteq L(G)$ by induction. First we have $\left(\Sigma \cup \Sigma^{2}\right) \cap D(1) \subseteq L(G)$. Now assume that $\left(\Sigma \cup \Sigma^{2} \cup \ldots \cup\right.$ $\left.\Sigma^{n}\right) \cap D(1) \subseteq L(G)$ for some $n \geq 2$. Let $u \in \Sigma^{n+1} \cap D(1)$. Consider the following two cases.

(Case 1) $u=x a y$ for $x, y \in \Sigma^{+}, a \in \Sigma$ with $|x|=|y|$.

(Case 2) $u=x a b y$ for $x, y \in \Sigma^{+}, a, b \in \Sigma$ with $|x|=|y|$.

Since $|x y| \leq n$, it follows that $x y \in D(1)$ by Lemma 7 , in either case. Then $\alpha \Rightarrow^{*} x y \Rightarrow u$ for some $\alpha \in A$. We have $u \in L(G)$. Thus $D(1) \subseteq L(G)$.:

\section{References}

[1] C.-M. Fan, H.J. Shyr, and S.S. Yu, "d-words and d-languages," Acta Informatica, vol.35, pp.709-727, 1998.

[2] P. Dömösi, S. Horvath, and M. Ito, "On the connection between formal languages and primitive words," Proc. First Session on Scientific Communication, University of Oradea, Romania, June 1991, Analele University of Oradea, Fasc, Mat., pp.59-67, 1991.

[3] P. Dömösi, S. Horvath, M. Ito, L. Kazonyi, and M. Katsura, "Formal languages consisting of primitive words," Lect. Note Comput. Sci., vol.710, pp.194-203, 1993.

[4] P. Dömösi, M. Ito, and S. Marcus, "Marcus contextual languages consisting of primitive words," Discrete Mathematics, vol.308, pp.4877-4881, 2008.

[5] S.C. Hsu, M. Ito, and H.J. Shyr, "Some properties of overlapping order and related languages," Soochow Journal of Mathematics, vol.15, pp.29-45, 1989.

[6] L. Kaszonyi and M. Katsura, "On the context-freeness of class of primitive words," Publ. Math. Debrecen, vol.51, nos.1-2, pp.1-11, 1997.

[7] I. Kataoka and T. Moriya, "d-primitive words and D(1)-concatenated words,” IEICE Trans. Inf. \& Syst., vol.E92-D, no.8, pp.1557-1579, Aug. 2009.

[8] T. Moriya and I. Kataoka, "On non-overlapping words," IEICE Trans. Inf. \& Syst., vol.E94-D, no.3, pp.707-709, March 2011.

[9] G. Păun, Marcus Contextual Grammars, Kluwer Academic Publishers, Dordrecht, Boston, London, 1997.

[10] H.J. Shyr, Free monoids and languages, Hon Min Book Company, Taichung, Taiwan, 2001.

[11] Z. Li and Y.S. Tsai, "Three-element codes with one d-primitive word," Acta Informatica, vol.41, nos.2-3, pp.171-180, 2004. 